\title{
Development of Automatic Bathtub Washer
}

\author{
Kohei Shinyamada ${ }^{\mathrm{a}}$, Takumi Saruhashi ${ }^{\mathrm{a}}$, Yuhki Kitazono ${ }^{\mathrm{a},{ }^{*}}$

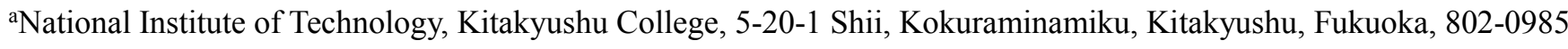 \\ Japan
}

*Corresponding Author: kitazono@kct.ac.jp

\begin{abstract}
The housework is a daily job, and there are many people feeling that cleaning a bathtub is particularly a hard task. According to a certain investigation, the first place of the housework that we want to automate is cleaning a bathtub, and the automation of cleaning a bathtub is needed. Therefore, I thought that the automation would be possible if I developed the bathtub washing machine which is attached the rotating brush to the arm that works along the plane of the bathtub. I developed a basic structure. By utilizing this, we can hope that everyday cleaning a bathtub will be easily, and the burden of the household chores will be reduced.
\end{abstract}

Keywords: Bathtub, Cleaning, Robot.

\section{Introduction}

The housework is a daily job, and it is particularly a hard task. Therefore, the automation of housework is needed. According to "The Questionnaire Survey about Housework" that was investigated by O-uccino, Inc., the survey for 813 married women who live in the metropolitan area from 20 to 59 years old says that the first place of the housework that we want to automate is cleaning a bathtub (54.5\%) shown in Fig. $1^{(1)}$. Moreover, the cleaning a bathtub is said to be particularly burdensome to the body in the housework. When we clean, we need squatting down or half-sitting, which is a burdensome position. Cleaning a bathtub is a burden to waist and a hard job for people who have backache or the elderly. In addition, a bathroom is the place, which has the elderly, can fall down in a bathroom. These make it clear that automation of the cleaning a bathtub is particularly demanded.

Now, Noritz Corporation sells "Osoji-Yokuso" (Osoji means cleaning in English, and Yokuso means bathtub in English.). This product injects the detergent and washes the

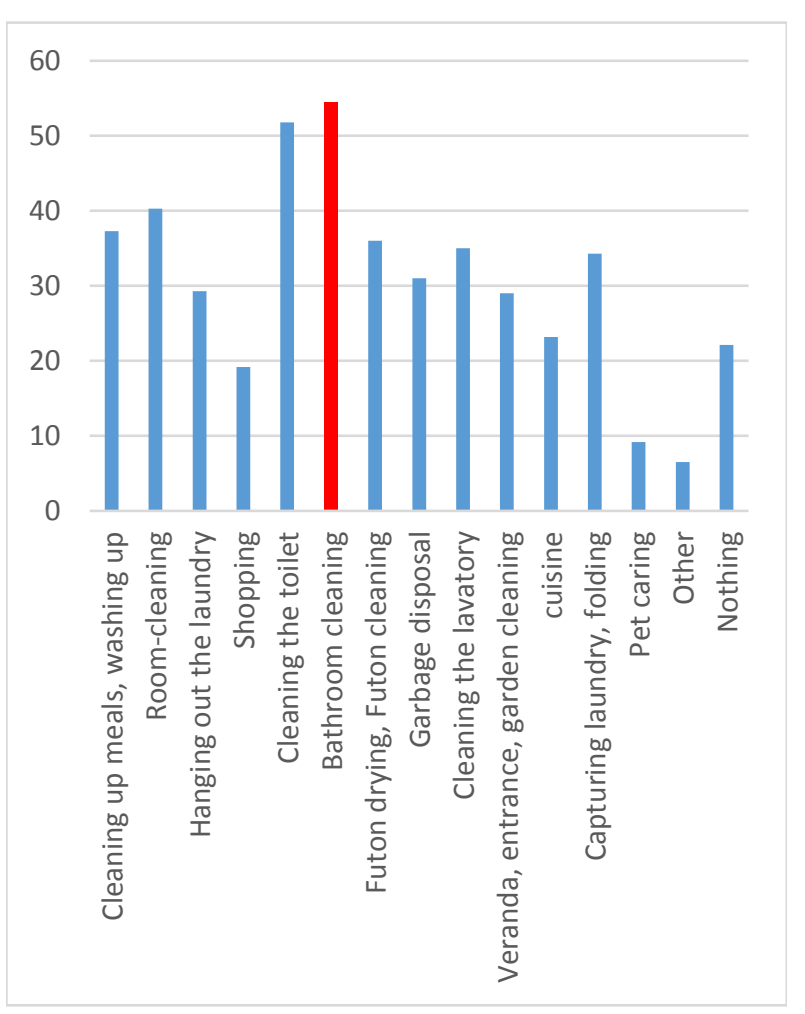

Fig. 1. Investigation result.

bathtub with hot water. However, this product has some problems. First, it has a limit to remove dirt by a jet only with the detergent and the current of water, and stuck dirt may not be removed. Next, this device is a built-in function. Therefore, it is impossible that the device is installed on existing bathtub. It takes time and costs a lot to remodel a bathtub.

In addition, there is a tool to support the cleaning a bathtub, that is an electric bath polisher. The product has the pole, which has an electric rotating brush on top. If we press it to the bathtub, we can clean that part. Undoubtedly, the cleaning becomes easy. However it is necessary to move the device by hand, and it cannot be said that it automates 
cleaning a bathtub.

Therefore, we will develop the basic structure of cleaning a bathtub machine that automatically scrubs the bathtub using an arm and a rotating brush in order to solve these problems. The device shall be attachable to an existing bathtub. Furthermore, it is possible automatically to clean along the flat surface of the bathtub, inject a detergent and wash the bathtub in water by improving this system.

\section{Structure}

\subsection{The entire structure}

An overview of the whole device is shown in Fig. 2. The structure of each part is shown in a later section.

\subsection{Arm}

Here, the structure of the arm for cleaning the bathtub will be described.

\subsubsection{Rotational mechanism in the horizontal direction}

A picture of the equipment and the bathtub from above is shown in Fig. 3. As shown in the figure, a lateral rotation mechanism is indispensable in order to move the arm along the bathtub.

A servomotor (KONDO KRS - 2552 RHC ICS) is used for this mechanism. A servomotor can specify angle, and it is often used positioning ${ }^{(2-4)}$. The arm and the brush are rotated in the lateral direction by rotating the motor.

\subsubsection{Rotating mechanism in the vertical direction}

A picture of the equipment and the bathtub from the front is shown in Fig. 4. As shown in the figure, a longitudinal rotation mechanism is indispensable in order to move the arm along the bathtub.

A power window motor (manufactured by MABUCHI MOTOR CO., LTD.) is used for this mechanism. By rotating the motor, the arm and rotating brush in the vertical direction.

\subsubsection{Expansion and contraction mechanism}

The structure is shown in Fig. 5. Even if it is rotated in the longitudinal and lateral directions, it is indispensable to adjust the length of the arm in order to move along the plane of the bathtub.

We use two pipes with different thickness, ball screws, and DC motors (Tamiya geared motor) for this mechanism. Store a thin pipe in a thick pipe. Attach a ball screw to the thin pipe and connect it to the DC motor. The ball screw

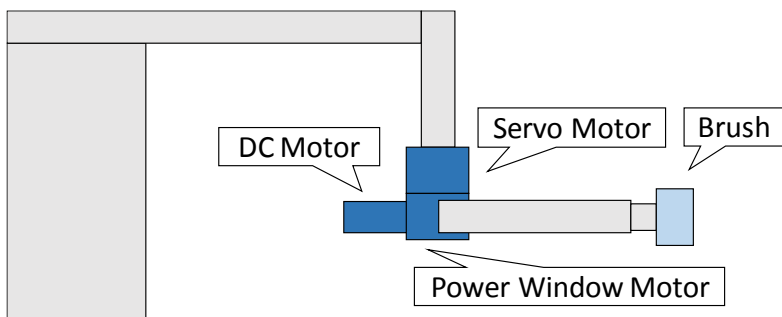

Fig. 2. Photo of equipment.

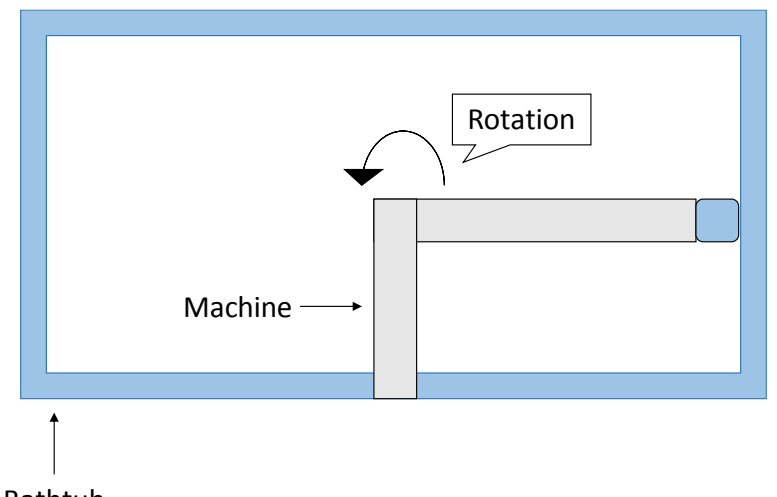

Bathtub

Fig. 3. A view of the equipment and the bathtub from above.

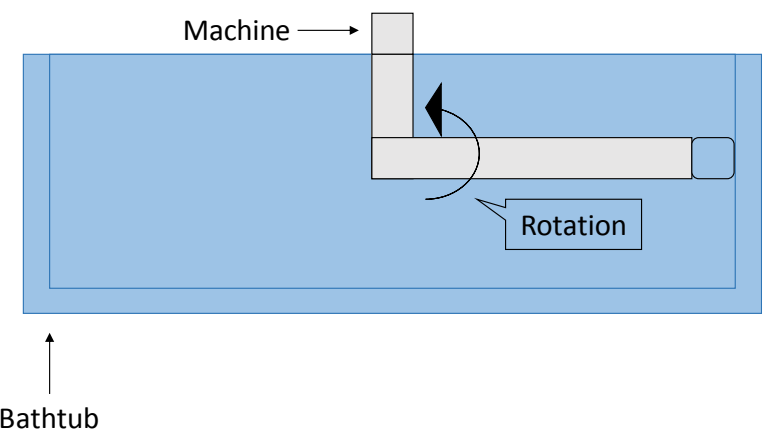

Fig. 4. Front view of the equipment and bathtub.

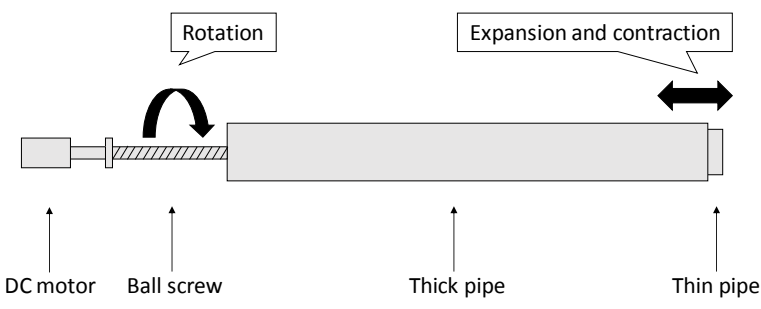

Fig. 5. Expansion and contraction mechanism. 


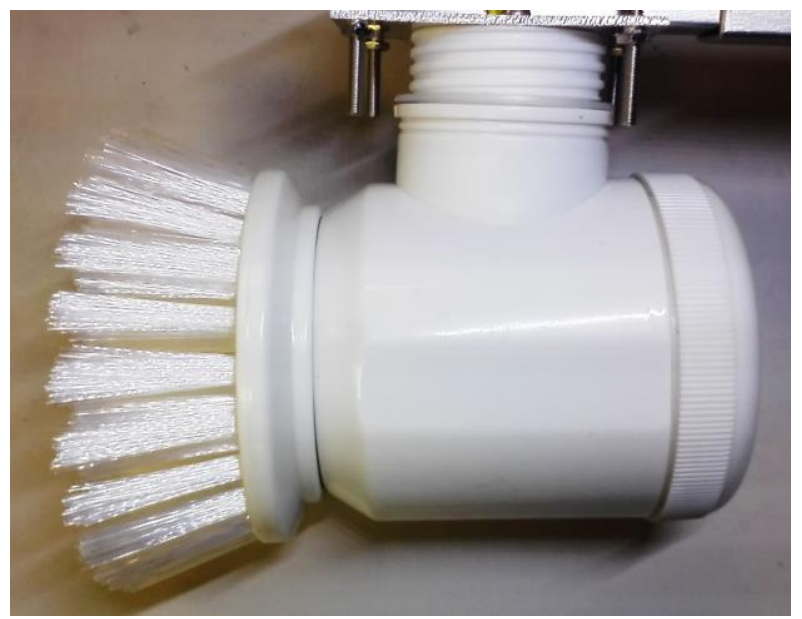

Fig. 6. Rotating brush.

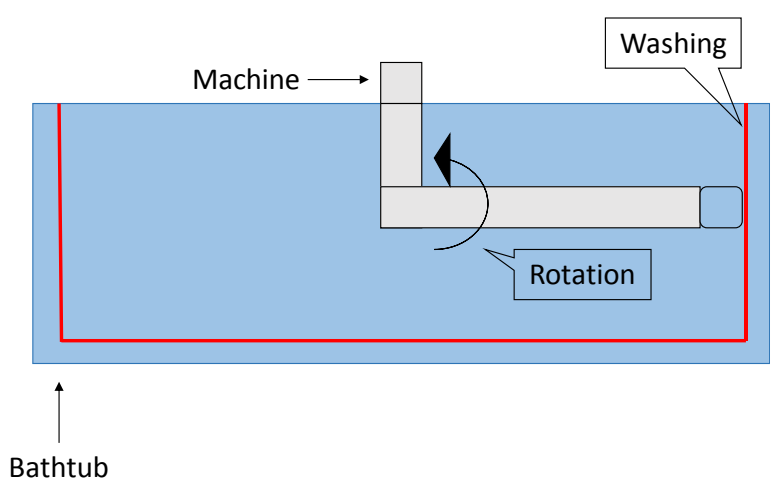

Fig. 7. Washing.

rotates, and it enables the arm to expand and contract by rotating the $\mathrm{DC}$ motor.

\subsection{Rotating brush}

A brush attached to the tip of the arm for cleaning is shown in Fig. 6.

Cleaning is possible by rotating this brush on a bathtub.

\subsection{Control}

Arduino (MEGA 2560) is used to control each motor. The motor driver used in the DC motor and the power window is VNH 2 SP 30. Programming is done to achieve the desired operation.

\section{Action}

The operation of the device will be described in this section. Activate the device and rotate the rotating brush. Then extend the arm to the bottom of the bathtub. Rotate the power window to clean it vertically. The motor of the expansion mechanism part is rotated at the same time in order to make it along the plane of the bathtub. It is possible to wash the bathtub in a V-shaped form (Fig. 7). Next, the servomotor is rotated to perform the lateral cleaning. Cleaning of the U-shaped character turns. The entire bathtub can be cleaned by repeating this operation.

\section{Experiment}

We describe the experiments of the equipment.

Experiments are carried out in each part, and lastly united.

\subsection{Rotational mechanism in the horizontal direction}

We connected the servo to Arduino and programed it to stop to some extent in order to check the lateral rotation motion. As a result, we were able to successfully overturn and determined the position.

\subsection{Rotating mechanism in the vertical direction}

The power window motor was connected to a constant voltage source in order to check the rotational motion in the vertical direction. As a result, it rotated in a good condition.

Also, power window motor was controled with a motor driver in order to perform the stable operation. However, the signal input of the motor driver was added from Arduino. This allowed Arduino to control the direction and speed of the motor.

\subsection{Expansion and contraction mechanism}

The DC motor rotated normally and expanded / contracted in order to confirm the operation of the expansion / contraction mechanism by connecting the constant voltage source to the terminal of the DC motor.

In addition, the DC motor was controlled with a motor driver in order to perform the stable operation. However, the signal input of the motor driver was added from Arduino. This allowed Arduino to control the direction and speed of the motor.

\subsection{Rotating brush}

The brush was rotated in a good condition by connecting the constant voltage source to the terminal in order to check the operation of the rotating brush

In addition, the terminal of the rotating brush was connected with Arduino in order to be able to control by Arduino. As a result, it was possible to control the turning brush ON / OFF of the rush from Arduino.

\subsection{The entire device}

In 4.1 to 4.4, the operation of each part was confirmed. Here, we checked the operation when they were integrated. A lithium ion battery was used as the power source of the 
entire device. The operation described in 3 could be successful.

\section{Conclusions}

By applying this device, it can be expected that the burden of daily housekeeping such as cleaning a bathtub will be reduced. Since this device has only a basic structure, fully automated bathtub cleaning system has not been realized. A distance sensor must be mounted and the arm must be expanded and contracted based on that value in order to move the arm along the plane of the bathtub. In addition, it is necessary for an electric pump to inject the detergent and wash in water in order to achieve the complete automation.

\section{References}

(1) O-uccino, Inc. : “The a Questionnaire Survey about Housework", 2015

(2) Toyokazu Uchida, Akihito Ito, Tatsuya Oshima, amd Nobuyuki Furuya : "Positioning System with Backlash Compensation by Twin Motor Cooperative Control", The Japan Society of Mechanical Engineers, Part C 77(778), pp. 2280-2289, 2011

(3) Toyokazu Uchida, Akihito Ito, Toshiya Kitamura, amd Nobuyuki Furuya : "Positioning system with backlash compensation by twin motor cooperative control (Evaluation of rectilinear motion mechanism installed planetary gear speed reducer)", The Japan Society of Mechanical Engineers, 80(814), DR0162-DR0162, 2014

(4) Tomonori Mashimo, Kiyoshi Ohishi, and Hideo Dohmeki : "High Speed Positioning Method for Servo Motor Considering Friction Load Torque", The transactions of the Institute of Electrical Engineers of Japan. D, A publication of Industry Applications Society 124(7), pp. 666-673, 2004-07-01 